\title{
Cognitive Interference Alignment between Small Cells and a Macrocell
}

\author{
Symeon Chatzinotas*, Björn Ottersten ${ }^{* \dagger}$ \\ ${ }^{*}$ SnT - securityandtrust.lu, University of Luxembourg, Email: \{Symeon.Chatzinotas, Bjorn.Ottersten $\} @$ uni.lu \\ ${ }^{\dagger}$ Royal Institute of Technology (KTH), Sweden, Email: bjorn.ottersten@ee.kth.se
}

\begin{abstract}
Small cells have attracted large interest lately in the research community, mainly due to easy and quick deployment. In addition, a large number of small cells is in general more energy efficient than macrocells since less power is required to combat path loss and get across the wireless medium. However, in current network configurations these two types of cells have to coexist over the same spectrum because existing cellular systems are mainly based on macrocells and additional bandwidth for small cell deployments is scarce and too expensive to acquire. In this context, this contribution investigates an underlay cognitive communication technique which exploits interference alignment across multiple antennas in order to mitigate the interference of small cell User Terminals (UTs) towards the macrocell Base Station (BS). More specifically, three techniques for aligning interference are investigated, namely static, coordinated and uncoordinated. The system performance is evaluated and compared based on sum-rate capacity, primary rate protection ratio and primary to secondary rate ratio.
\end{abstract}

\section{INTRODUCTION}

Since the infancy of cellular architectures, it has been well established that denser deployments can provide higher capacity and availability. However, during the early cellular deployments the technology was not mature enough to allow for large numbers of inexpensive radio stations. Nowadays, the technology is available but dense cellular networks (small cells) have to coexist with traditional macrocells due to wireless spectrum scarcity. This need has lead to the inspiration of cognitive communications which allow for the coexistence of two systems, primary and secondary, over the same frequency resources. The operation of the primary network usually follows a well established standard and should not be degraded, while the secondary network should employ advanced communication techniques to exploit underutilized dimensions in the signal space. In this direction, this work investigates an underlay cognitive communication technique which exploits uplink interference alignment in order to mitigate the interference of small cell User Terminals (UTs) towards the macrocell Base Station (BS). Small cells are assumed to be interconnected through fiber (Radio over Fiber) to a central receiver which performs multicell joint decoding of the received signals. The proposed technique is compared to a passive transmission technique which allows for cochannel interference, as well as to a resource splitting approach. The performance metrics of interest studied in this work are the ergodic-sum rate capacity over an asymmetric MIMO Ricean channel, primary rate protection ratio and primary to secondary rate ratio.
The remainder of this paper is structured as follows: Section II reviews in detail prior work in the areas of small cells and Interference Alignment (IA). Section III describes the system and channel modeling, while section IV describes the proposed interference alignment technique and the considered performance metrics. Section $\mathrm{V}$ evaluates the effect of various parameters on the system performance. Section VI concludes the paper.

\section{A. Notation}

Throughout the formulations of this paper, $\mathbb{E}[\cdot]$ denotes the expectation, $(\cdot)^{\dagger}$ denotes the conjugate transpose matrix, $(\cdot)^{T}$ denotes the transpose matrix, $\odot$ denotes the Hadamard product and $\otimes$ denotes the Kronecker product. $\mathbf{I}_{n}$ denotes a $n \times n$ identity matrix, $\mathbb{I}_{n \times m}$ a $n \times m$ matrix of ones, $\mathbf{0}$ a zero matrix and $\mathbf{G}_{n \times m}$ a $n \times m$ Gaussian matrix.

\section{Preliminaries \& Related Work}

This section introduces the concepts of small cells, multicell joint decoding (MJD) and interference alignment. Subsequently, relevant literature is reviewed.

\section{A. Multicell Joint Decoding}

The paradigm of global MJD (also known as BS cooperation) was initially proposed in two seminal papers [1], [2]. The main assumption is the existence of a central processor which is interconnected to all the BSs through a backhaul of wideband, delayless and error-free links. In addition, the central processor is assumed to have perfect Channel State Information (CSI) about all the wireless links of the system. These assumptions enable the central processor to jointly decode all the UTs of the system, rendering the concept of intercell interference void. In this context, it was demonstrated in [3] that Rayleigh fading promotes multiuser diversity which is beneficial for the ergodic capacity performance. Subsequently, realistic path-loss models and user distribution were investigated in [4], [5] providing closed-form capacity expressions based on the cell size, path loss exponent and user spatial p.d.f. The beneficial effect of MIMO links was established in [6], [7], where a linear scaling with the number of BS antennas was proven.

\section{B. Interference Alignment}

IA has been shown to achieve the degrees of freedom for a range of interference channels [8]-[10]. Its principle 
is based on aligning the interference on a signal subspace with respect to the non-intended receiver, so that it can be easily filtered out by sacrificing some signal dimensions. The advantage is that this alignment does not affect the randomness of the signals and the available dimensions with respect to the intended receiver. The disadvantage is that the filtering at the non-intended receiver removes the signal energy in the interference subspace and reduces the multiplexing gain. The fundamental assumptions which render interference alignment feasible are that there are multiple available dimensions (space, frequency, time or code) and that the transmitter is aware of the CSI towards the non-intended receiver. The exact number of needed dimensions and the precoding vectors to achieve interference alignment are rather cumbersome to compute, but a number of approaches have been presented in the literature towards this end [11]-[13]. It should be noted that IA can be classified as an underlay cognitive technique [14] since it deals with interference mitigation towards the primary system in frequency coexistence scenarios.

\section{IA for Cellular Systems}

Interference alignment has been also investigated in the context of cellular networks, showing that it can effectively suppress cochannel interference [13], [15]. More specifically, the downlink of OFDMA cellular network with clustered multicell processing is considered in [13], where interference alignment is employed to suppress intracluster interference while intercluster interference has to be tolerated as noise. In addition, authors in [15] consider the uplink of a limited-size cellular system without MJD, showing that the interferencefree degrees of freedom (dofs) can be achieved as the number of UTs grows large. In the same context, authors in [16] employ IA as an uplink interference mitigation technique amongst cooperating BS clusters for Rayleigh channels. Coming back to small cells, the work in [17] extends [16] by assuming clusters of small cells which dictate the use of a Ricean channel. Finally, the authors in [18] propose Vandermondesubspace Frequency Division Multiplexing for the downlink in order to null out the interference of small cells towards primary macro users.

\section{System MODEL}

Let us consider a coverage area where a single macrocell operates receiving signals from a set of primary users. A number of small cells $(N)$ operate over the same coverage area receiving signals from a set of secondary users. Furthermore, the small cells are able of cooperating through a broadband backhaul (e.g. Radio over Fiber) and jointly decoding the received signals. After scheduling, we consider that for a single slot one macro UT and $N$ small cell UTs are transmitting simultaneously over a common set of frequencies (Fig. 1). Since the macrocell system is primary, interference coming from the small cell UTs has to be suppressed. On the other hand, the interference of the macro UT towards the small Access Points (APs) has to be tolerated as the small cell system is secondary. All receivers and transmitters are equipped with

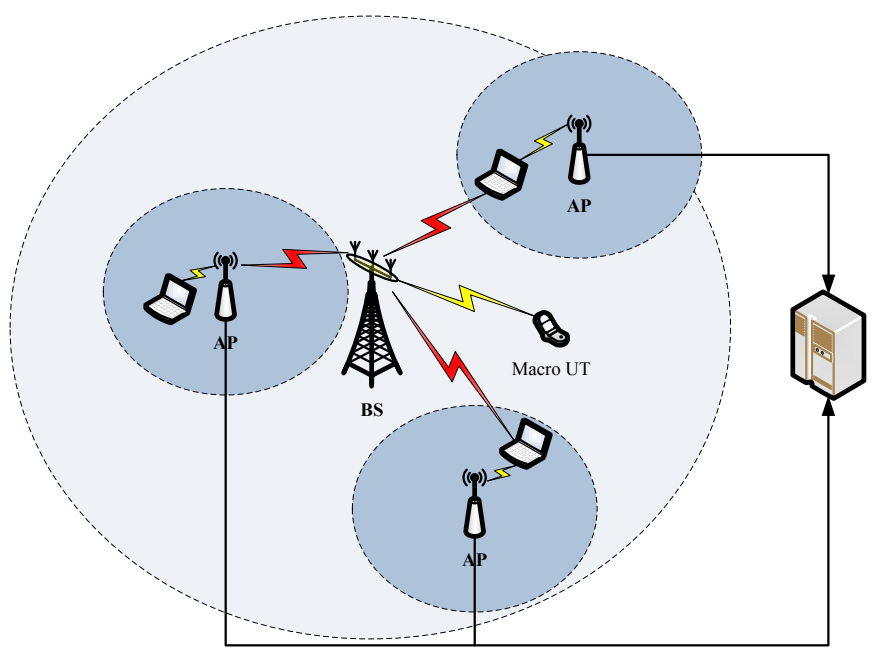

Fig. 1. Graphical representation of the considered cellular system model.

$M$ multiple antennas. More specifically, the macro UT has $M$ antennas, while the BS, small cell UTs and AP have $L=M+1$ antennas. In order to suppress the interference caused by the small cell UTs, we assume that they have Channel State Information (CSI) towards the macro BS. This CSI can be easily measured if the small cell UTs are aware of the macrocell pilot signals. In addition, there is a predefined vector $\mathbf{v}$ which is known by both small cell UTs and macro $\mathrm{BS}^{1}$. The idea behind cognitive interference alignment is to employ precoding at the small cell UTs so that the received secondary signals at the macro BS are all aligned across vector v. This way, interference can be filtered out by sacrificing one spatial degree of freedom and part of the received energy. However, after filtering the signal is interference-free and can be decoded just as in a conventional macrocellular system. The term cognitive comes from the fact that small cell UTs have to be aware of the CSI and vector $\mathbf{v}$ to perform the precoding. On the other hand, the macro BS needs only to perform spatial filtering over vector $\mathbf{v}$ and no additional awareness or intelligence is needed.

\section{A. Signal Model}

The received signal at the macro BS (primary link) is:

$$
\mathbf{y}_{1}=\mathbf{H} \mathbf{x}+\sum_{i=1}^{N} \mathbf{F}_{i} \mathbf{x}_{i}+\mathbf{z}_{1},
$$

where $\mathbf{y}_{1}$ is the $L \times 1$ received symbol vector, $\mathbf{x}$ is the $M \times$ 1 transmitted symbol vector from the macro UT, $\mathbf{x}_{i}$ is the $L \times 1$ transmitted symbol vector from the $i$ th small-cell UT and $\mathbf{z}_{1}$ is the receiver noise. All inputs $\mathbf{x}, \mathbf{x}_{i}$ are Gaussian and obey the following sum power constraints: $\mathbb{E}\left[\mathbf{x}^{\dagger} \mathbf{x}\right] \leq \gamma M$ and $\mathbb{E}\left[\mathbf{x}_{i}^{\dagger} \mathbf{x}_{i}\right] \leq \gamma L$. The $L \times M$ matrix $\mathbf{H}$ represents the channel gains between BS and macro UT, while the $L \times L$ matrix $\mathbf{F}_{i}$ represents the channel gains between BS and $i$ th smallcell UT. To simplify notations we group all $\mathbf{F}_{i}$ into a single

\footnotetext{
${ }^{1}$ Depending on how $\mathbf{v}$ is calculated, we define later on three IA techniques: static, coordinated, uncoordinated.
} 
$L \times N L$ matrix $\mathbf{F}=\left[\mathbf{F}_{1} \ldots \mathbf{F}_{N}\right]$. The received signal at the joint processor of the APs (secondary link) is:

$$
\mathbf{y}_{2}=\sum_{i=1}^{N} \tilde{\mathbf{F}}_{i} \mathbf{x}_{i}+\tilde{\mathbf{H}} \mathbf{x}+\mathbf{z}_{2},
$$

where $\mathbf{y}_{2}$ is the $N L \times 1$ received symbol vector and $\mathbf{z}_{2}$ is the receiver noise. The $N L \times M$ channel matrix $\tilde{\mathbf{H}}$ represents the channel gains between all APs and macro UT, while the $N L \times$ $L$ channel matrix $\tilde{\mathbf{F}}_{i}$ represents the channel gains between all APs and the $i$ th small-cell UT. To simplify notations we group all $\tilde{\mathbf{F}}_{i}$ into a single $N L \times N L$ matrix $\tilde{\mathbf{F}}=\left[\tilde{\mathbf{F}}_{1} \ldots \tilde{\mathbf{F}}_{N}\right]$.

\section{B. Channel Model}

The considered channel model is based on a Rayleigh channel whose power is scaled according to a power-law path loss model (i.e. asymmetric power levels). More specifically,

$$
\mathbf{H}=\alpha \mathbf{G}
$$

where $\alpha$ is the path loss coefficient between BS and macro UT and $\mathbf{G}$ is a random matrix with complex circularly symmetric (c.c.s.) independent identically distributed (i.i.d.) elements representing Rayleigh fading coefficients. Similarly,

$$
\mathbf{F}_{i}=\alpha_{i} \mathbf{J}_{i}
$$

where $\alpha_{i}$ is the path loss coefficient between BS and $i$ th smallcell UT. As a result,

$$
\mathbf{F}=\left(\boldsymbol{\alpha}^{T} \otimes \mathbb{I}_{L \times L}\right) \odot \mathbf{J},
$$

with $\boldsymbol{\alpha}=\left[\alpha_{1} \ldots \alpha_{N}\right]^{T}$. In addition,

$$
\tilde{\mathbf{H}}=\left(\boldsymbol{\beta} \otimes \mathbb{I}_{L \times M}\right) \odot \tilde{\mathbf{G}}
$$

where $\boldsymbol{\beta}=\left[\beta_{1} \ldots \beta_{N}\right]^{T}$ includes path loss coefficients between all APs and macro UT. Similarly,

$$
\tilde{\mathbf{F}}_{i}=\left(\boldsymbol{\beta}_{i} \otimes \mathbb{I}_{L \times L}\right) \odot \tilde{\mathbf{J}}_{i},
$$

where $\boldsymbol{\beta}_{i}$ contains the path loss coefficient between all APs and the $i$ th small-cell UT. As a result,

$$
\tilde{\mathbf{F}}=\left(\mathbf{B} \otimes \mathbb{I}_{L \times L}\right) \odot \mathbf{J},
$$

with $\mathbf{B}=\left[\boldsymbol{\beta}_{1} \ldots \boldsymbol{\beta}_{N}\right]$. It should be noted that all Line of Sight (LoS) matrices $\mathbf{L}, \mathbf{M}_{i}, \tilde{\mathbf{L}}, \tilde{\mathbf{M}}_{i}$ are considered unit rank due to the high correlation in the LoS signal path. In order to calculate the path loss coefficients, a power-law path loss model is considered while macro and small-cell UTs are uniformly distributed within the coverage area of BS and APs respectively (Fig. 1). The APs are also uniformly distributed within the coverage area of the BS. The ergodic metrics are evaluated by averaging over a large number of channel realizations and positions.

\section{System Performance}

This section studies the capacity expressions for the considered transmission techniques and subsequently describes three methods for determining the alignment direction $\mathbf{v}$.

\section{A. Capacity Expressions}

1) Primary Only: This case corresponds to current frequency allocations, according to which each band is allocated only to a primary system. For the considered system the primary throughput can be written as:

$$
\mathrm{C}_{p o}=\mathbb{E}\left[\log \operatorname{det}\left(\mathbf{I}_{L}+\frac{\gamma_{m c}}{M} \mathbf{H} \mathbf{H}^{\dagger}\right)\right]
$$

2) Interference-limited: Assuming no interference mitigation and uniform power allocation across the multiple transmit antennas of the UTs, the primary throughput can be written as:

$$
\mathrm{C}_{m c}=\mathbb{E}\left[\log \operatorname{det}\left(\mathbf{I}_{L}+\frac{\gamma_{m c}}{M} \mathbf{H} \mathbf{H}^{\dagger}\left(\mathbf{I}_{L}+\frac{\gamma_{s c}}{L} \mathbf{F} \mathbf{F}^{\dagger}\right)^{-1}\right)\right]
$$

while the secondary throughput as:

$$
\mathrm{C}_{s c}=\mathbb{E}\left[\log \operatorname{det}\left(\mathbf{I}_{N L}+\frac{\gamma_{s c}}{L} \tilde{\mathbf{F}} \tilde{\mathbf{F}}^{\dagger}\left(\mathbf{I}_{N L}+\frac{\gamma_{m c}}{M} \tilde{\mathbf{H}} \tilde{\mathbf{H}}^{\dagger}\right)^{-1}\right)\right]
$$

In both cases the second term represents cochannel interference.

3) Resource Splitting: This case assumes that the available bandwidth is split in two in order to allow the interferencefree operation of both primary and secondary. Although this is an impractical scenario $^{2}$, we consider it for the sake of completeness. The primary throughput can be written as:

$$
\hat{\mathrm{C}}_{p r}=\frac{1}{2} \mathbb{E}\left[\log \operatorname{det}\left(\mathbf{I}_{L}+\frac{2 \gamma_{m c}}{M} \mathbf{H} \mathbf{H}^{\dagger}\right)\right]
$$

while the secondary throughput as:

$$
\hat{\mathrm{C}}_{s r}=\frac{1}{2} \mathbb{E}\left[\log \operatorname{det}\left(\mathbf{I}_{N L}+\frac{2 \gamma_{s c}}{L} \tilde{\mathbf{F}} \tilde{\mathbf{F}}^{\dagger}\right)\right]
$$

4) Interference Alignment: By employing interference alignment towards the primary macrocell, the interference can be filtered out, resulting in the following primary throughput:

$$
\overline{\mathrm{C}}_{m c}=\mathbb{E}\left[\log \operatorname{det}\left(\mathbf{I}_{M}+\frac{\gamma_{m c}}{M} \overline{\mathbf{H}} \overline{\mathbf{H}}^{\dagger}\right)\right],
$$

where $\overline{\mathbf{H}}$ is the equivalent channel matrix after IA filtering. For the secondary small cells, the interference has to be tolerated and thus the secondary throughput can be written as:

$$
\overline{\mathrm{C}}_{s c}=\mathbb{E}\left[\log \operatorname{det}\left(\mathbf{I}_{N L}+\frac{\gamma_{s c}}{L} \overline{\mathbf{F}} \overline{\mathbf{F}}^{\dagger}\left(\mathbf{I}_{N L}+\frac{\gamma_{m c}}{M} \tilde{\mathbf{H}} \tilde{\mathbf{H}}^{\dagger}\right)^{-1}\right)\right]
$$

where $\overline{\mathbf{F}}$ is the equivalent channel matrix including precoding.

\footnotetext{
${ }^{2}$ The primary system would have to concede half of its spectrum.
} 


\section{B. Interference Alignment \& Filtering}

Let us assume a $L \times 1$ non-zero reference vector $\mathbf{v}$ along which the interference should be aligned. It should be noted that small cell UTs are assumed to know the alignment direction $^{3} \mathbf{v}$ and to have perfect own CSI about the channel coefficients $\mathbf{F}_{i}$ towards the BS. In this context, the following precoding scheme is employed to align interference:

$$
\mathbf{x}_{i}=\mathbf{w}_{i} x_{i}=\left(\mathbf{F}_{i}\right)^{-1} \mathbf{v} v_{i} x_{i},
$$

where $\|\mathbf{v}\|^{2}=L$ and $\mathbb{E}\left[\mathbf{x}_{i}^{\dagger} \mathbf{x}_{i}\right] \leq L \gamma$. the scaling variable $v_{i}$ is needed to ensure that the input power constraint is not violated for each UT. This precoding results in unit multiplexing gain and is by no means the optimal IA scheme, but it serves as a tractable way of evaluating the IA performance. Following this approach, the cochannel interference can be expressed as:

$$
\sum_{i=1}^{N} \mathbf{F}_{i} \mathbf{x}_{i}=\sum_{i=1}^{N} \mathbf{F}_{i}\left(\mathbf{F}_{i}\right)^{-1} \mathbf{v} v_{i} x_{i}=\mathbf{v} \sum_{i=1}^{N} v_{i} x_{i} .
$$

It can be easily seen that interference has been aligned across the reference vector and it can be removed using a $M \times L$ zero-forcing filter $\mathbf{Q}$ designed so that $\mathbf{Q}$ is a truncated unitary matrix [9] and $\mathbf{Q v}=\mathbf{0}$. After filtering, the $M \times 1$ received signal vector at the $\mathrm{BS}$ can be expressed as:

$$
\overline{\mathbf{y}}_{1}=\overline{\mathbf{H}} \mathbf{x}+\overline{\mathbf{z}}_{1}
$$

where $\overline{\mathbf{H}}=\mathbf{Q H}$ is the $M \times M$ filtered channel matrix. Assuming that the system operates in high-SNR regime and is therefore interference limited, the effect of the AWGN noise colouring $\overline{\mathbf{z}}_{1}=\mathbf{Q} \mathbf{z}_{1}$ can be ignored, namely $\mathbb{E}\left[\overline{\mathbf{z}}_{1} \overline{\mathbf{z}}_{1}^{H}\right]=\mathbf{I}$. Furthermore, the received signal at the joint processor of the APs (secondary link) is:

$$
\overline{\mathbf{y}}_{2}=\sum_{i=1}^{N} \overline{\mathbf{F}}_{i} x_{i}+\tilde{\mathbf{H}} \mathbf{x}+\mathbf{z}_{2},
$$

where $\overline{\mathbf{F}}_{i}=\tilde{\mathbf{F}}_{i}\left(\mathbf{F}_{i}\right)^{-1} \mathbf{v} v_{i}$ are the equivalent $N L \times 1$ channel matrices including precoding. To simplify notations we group all $\overline{\mathbf{F}}_{i}$ into a single $N L \times N$ matrix $\overline{\mathbf{F}}=\left[\overline{\mathbf{F}}_{1} \ldots \overline{\mathbf{F}}_{N}\right]$.

Theorem 4.1: For $L=M$, IA is asymptotically optimal with number of antennas in rejecting secondary interference:

$$
\lim _{M \rightarrow \infty} \overline{\mathrm{C}}_{m c} \longrightarrow \lim _{M \rightarrow \infty} \mathrm{C}_{p o} .
$$

Proof: The asymptotic capacity of (14) can be written using the property $\operatorname{det}(\mathbf{I}+\gamma \mathbf{A B})=\operatorname{det}(\mathbf{I}+\gamma \mathbf{B A})$ as:

$$
\lim _{M \rightarrow \infty} \overline{\mathbf{C}}_{m c}=\lim _{M \rightarrow \infty} \mathbb{E}\left[\log \operatorname{det}\left(\mathbf{I}_{M}+\frac{\alpha^{2} \gamma_{m c}}{M} \mathbf{G}^{\dagger} \mathbf{Q}^{\dagger} \mathbf{Q G}\right)\right]
$$

The $M \times L$ truncated unitary matrix $\mathbf{Q}$ has $M$ unit singular values and therefore the matrix product $\mathbf{Q}^{\dagger} \mathbf{Q}$ has $M$ unit eigenvalues and a zero eigenvalue. Applying eigenvalue

\footnotetext{
${ }^{3}$ As discussed in the next subsection, the alignment direction for each group of UT could be predetermined or alternatively coordinated via signaling through the intended BS or the small cell cluster.
}

decomposition on $\mathbf{Q}^{\dagger} \mathbf{Q}$, the left and right eigenvectors can be eliminated due to unitarily invariance, while the zero eigenvalue removes one of the $n$ dimensions. The asymptotic eigenvalue distribution of $\mathbf{G G}^{\dagger}$ depends not on the matrix size, but only on the ratio of horizontal to vertical dimensions [4], [19]. As a result, the single missing dimensions becomes asymptotically insignificant with the number of antennas and the two capacities converge.

\section{Alignment Direction Selection and Filter Design}

In this section, we investigate various approaches for selecting the alignment direction $\mathbf{v}$ and designing the corresponding filter Q. Since these two operations are interdependent, they have to be jointly studied taking into account the level of coordination between the primary and secondary system.

1) Static approach: In this approach, the alignment direction is predefined and does not depend on the channel state. It can be seen that this is a quite static but also simple solution which assumes no coordination in the network. The disadvantage lies in the fact that the IA direction may be aligned with one of the strong eigenvectors of the random BSmacro UT channel and in this case a large amount of received power will be filtered out.

2) Coordinated approach: This approach entails that the selection of the alignment direction takes place at the BS and is subsequently communicated to the small UTs. It is assumed that the channel coherence time is adequate for the alignment direction to be fed back and used by small UTs. This is an egoistic approach since the BS dictates the behavior of the secondary UTs in order to maximize the performance of the primary system. In this context, the following optimization problem can be defined:

$$
[\mathbf{v}, \mathbf{Q}]=\arg \max _{\mathbf{v}, \mathbf{Q}} \overline{\mathrm{C}}_{m c}, \quad \text { s.t. } \mathbf{Q v}=0, \mathbf{Q} \mathbf{Q}^{\dagger}=1
$$

Now let $\overline{\mathbf{H}} \overline{\mathbf{H}}^{\dagger}=\mathbf{U} \boldsymbol{\Lambda} \mathbf{U}^{\dagger}$ be the eigenvalue decomposition of $\overline{\mathbf{H}} \overline{\mathbf{H}}^{\dagger}$ and $\boldsymbol{\lambda}\left(\overline{\mathbf{H}} \overline{\mathbf{H}}^{\dagger}\right)=\left[\lambda_{1} \ldots \lambda_{M}\right]$ are the $M$ ordered non-zero eigenvalues. The eigenvectors define an orthonormal space of the MIMO subchannels. In this direction, the optimal strategy is to select the eigenvector which corresponds to the weakest eigenvalue $\lambda_{1}$ as alignment direction ${ }^{4}$. The filter can be easily designed using the $\mathbf{M}$ dominant eigenvectors of $\mathbf{H H}^{\dagger}$.

Corollary 4.1: For $L=M+1$, coordinated IA is optimal in rejecting secondary interference:

$$
\overline{\mathrm{C}}_{m c}=\mathrm{C}_{p o} .
$$

Proof: Using eigenvalue decompositions and linear algebra calculations, it can be seen that $\mathbf{H}^{\dagger} \mathbf{Q}^{\dagger} \mathbf{Q H}$ and $\mathbf{H}^{\dagger} \mathbf{H}$ have identical eigenvalues due to the fact that $\mathbf{H}^{\dagger} \mathbf{H}$ and $\mathbf{Q}^{\dagger} \mathbf{Q}$ share an eigenvector.

\footnotetext{
${ }^{4}$ If transmit CSI available is available at the macro UT, the available power can be optimally allocated through waterfilling to the remaining subchannels in order to maximize the throughput
} 
TABLE I

PARAMETERS FOR CAPACITY RESULTS

\begin{tabular}{l|c|c|c} 
Parameter & Symbol & Value & Range \\
\hline Number of small cells & $N$ & & $1-10$ \\
Macro UT antennas & $M$ & 2 & \\
Small UT, BS, AP antennas & $L$ & 3 & \\
Macrocell Radius & $R_{m c}$ & $2 K m$ & \\
Small Cell Radius & $R_{s c}$ & $600 m$ & \\
Macro UT Transmit Power & $\gamma_{m c}$ & $1 W$ & \\
Small UT Transmit Power & $\gamma_{s c}$ & $250 m W$ & \\
Path loss exponent & $\eta$ & 3.5 & \\
Number of MC iterations & & $10^{3}$ & \\
\hline
\end{tabular}

3) Uncoordinated approach: This approach assumes that the primary and the secondary system do not coordinate. Furthermore, small UTs are aware of their CSI towards the BS but have no information about the CSI of the macro UT. In this context, the small UTs have no other option than selecting an alignment direction which maximizes the secondary throughput. Subsequently, the BS is responsible for sensing the alignment direction and applying the appropriate filter. In this context, the following optimization problem can be defined:

$$
[\mathbf{v}, \mathbf{Q}]=\arg \max _{\mathbf{v}, \mathbf{Q}}\left(\overline{\mathrm{C}}_{s c}\right), \text { s.t. } \mathbf{Q v}=0, \mathbf{Q Q}^{\dagger}=1 .
$$

Since the interference channel coefficients $\tilde{\mathbf{H}}$ are not known, we employ a simplified objective function:

$$
[\mathbf{v}, \mathbf{Q}]=\arg \max _{\mathbf{v}, \mathbf{Q}} \operatorname{trace}\left(\overline{\mathbf{F}} \overline{\mathbf{F}}^{\dagger}\right) \text {. }
$$

The variables $u_{i}$ cannot be analytically calculated, so a heuristic solution for this problem would be to select the strongest eigenvector of the equivalent channel covariance $\operatorname{matrix} \sum_{i=1}^{L} \tilde{\mathbf{F}}_{i}\left(\mathbf{F}_{i}^{-1}\right)\left(\mathbf{F}_{i}^{\dagger}\right)^{-1} \tilde{\mathbf{F}}_{i}^{\dagger}$.

\section{NumericAl RESUlts}

This section presents a number of numerical results in order to provide a comparative evaluation of the proposed technique. Table I presents an overview of the parameter values and ranges used for producing the numerical results of the figures.

\section{A. Performance Metrics}

In order to evaluate the system performance, three different metrics are considered. The system sum-rate capacity can be defined as:

$$
\mathrm{C}_{s y s}=\mathrm{C}_{m c}+\frac{\mathrm{C}_{s c}}{N}
$$

where $\mathrm{C}_{m c}$ is the capacity of the macrocell and $\mathrm{C}_{s c}$ is the sumrate of the small cells. Subsequently, the primary to secondary rate ratio is defined as:

$$
\operatorname{PSR}=\frac{\mathrm{C}_{m c}}{\mathrm{C}_{s c} / N}
$$

Finally, the primary rate protection ratio can be defined as:

$$
\mathrm{PR}=\frac{\mathrm{C}_{m c}}{\mathrm{C}_{p o}} .
$$

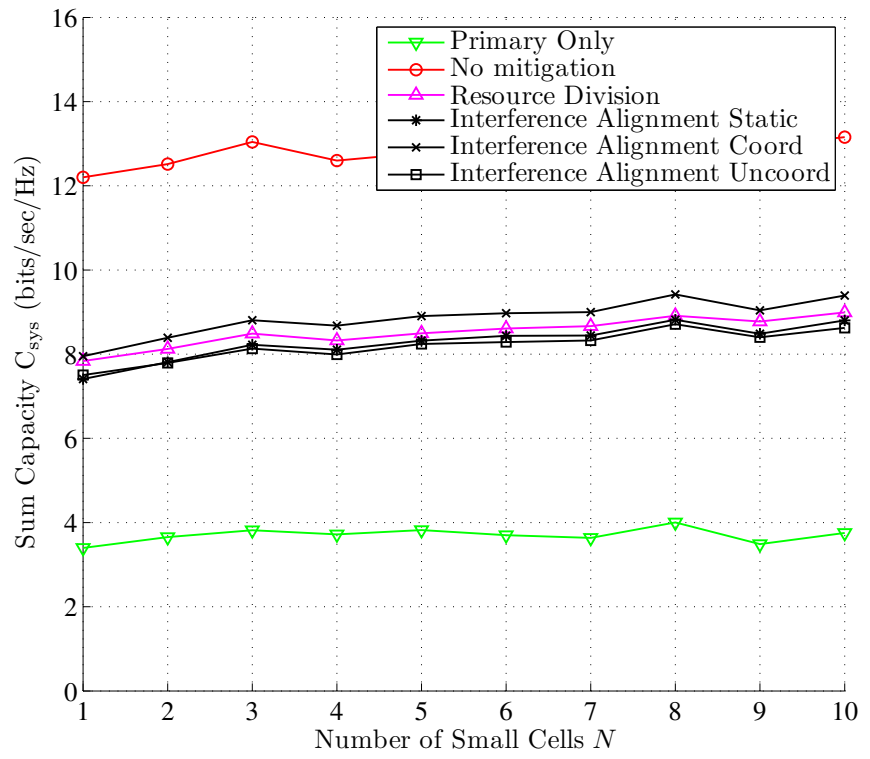

Fig. 2. Normalized system rate vs. number of small cells $N$.

\section{B. Results \& Discussion}

Figure 2 depicts the system rate and it can be seen that for all techniques it slowly increases with $N$. No-mitigation achieves a three-fold gain while other techniques a two-fold gain compared to primary only transmission. One could say that no-mitigation is promising but looking at the primary to secondary rate ratio (Figure 3) we can see that it does not perform well, especially for large $N$. In general, primary to secondary rate ratio decreases as more secondary small cells are included into the system. IA techniques have the best performance with the coordinated approach ranking first. This observation is supported and verified by the primary protection ratio (Figure 4). It should be especially noted that coordinated IA fully protects the primary rate as expected, while other IA techniques preserve roughly $70 \%$ and resource division $82 \%$ of the primary rate. Furthermore, all techniques except no-mitigation preserve a constant protection ratio with increasing $N$, while the performance of no-mitigation degrades monotonically.

\section{CONCLUSION}

This paper has investigated the cognitive coexistence of small cells and a macrocell equipped with MIMO transceivers. The primary only case was compared to coexistence with no mitigation, resource division and interference alignment. More specifically, three types of IA were considered depending on the level of network coordination and on whether it adapts to channel conditions. The different techniques were compared in terms of system rate, primary to secondary rate ratio and primary rate protection ratio. The highest system rate is achieved without interference mitigation but the primary protection degrades with the number of small cells. On the other hand, the coordinated IA perfectly protects the primary transmission while achieving the system rate of resource 


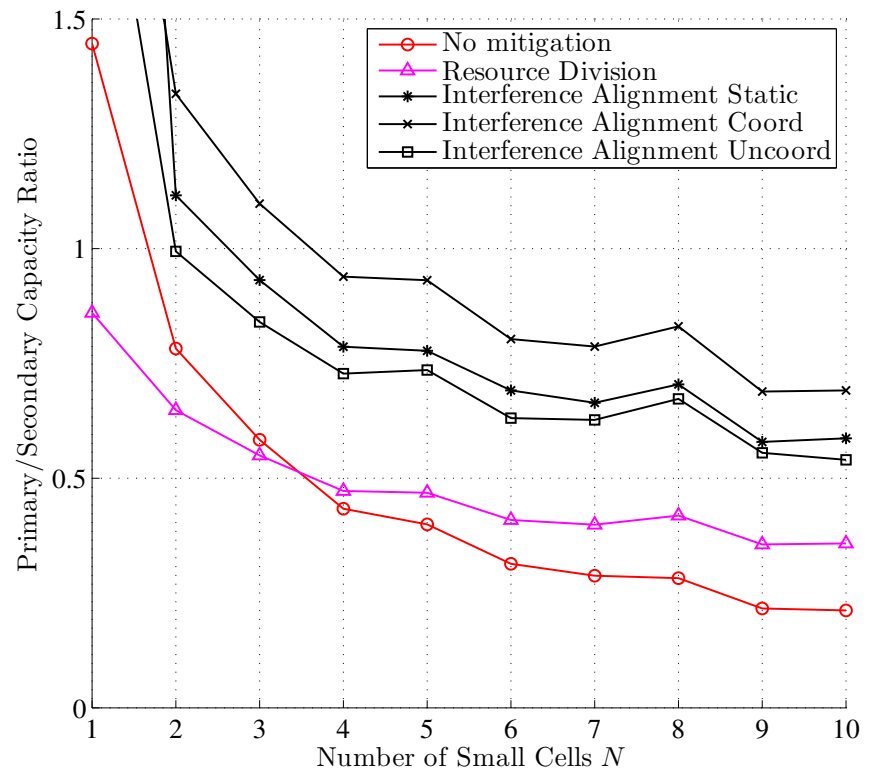

Fig. 3. Primary to secondary rate ratio vs. number of small cells $N$.

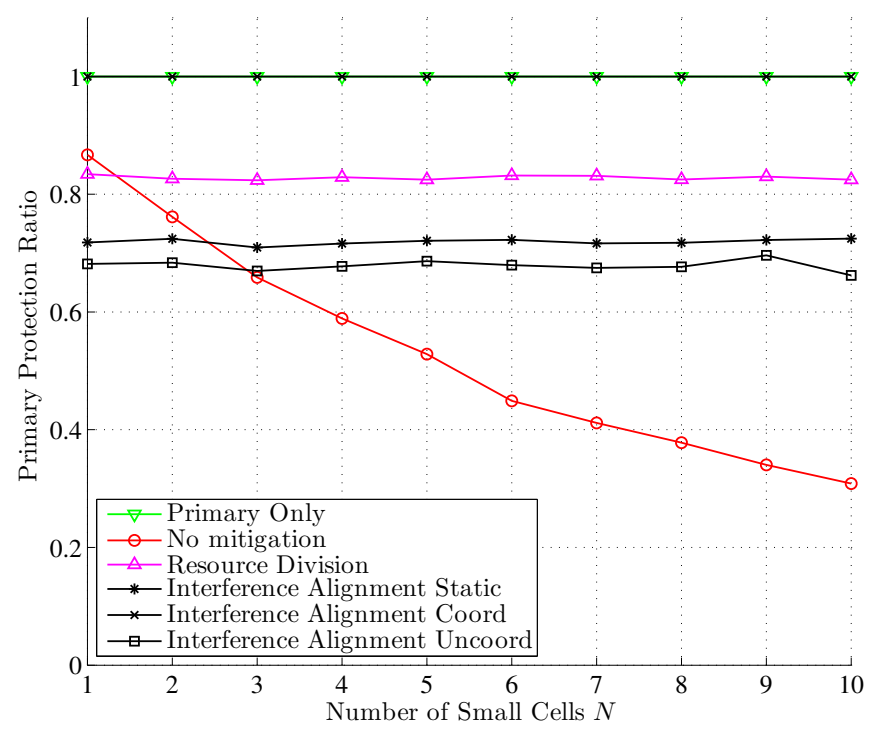

Fig. 4. Primary protection ratio vs. number of small cells $N$.

division. Future work will focus on finding closed-form expressions for the performance metrics through large system analysis and incorporating antenna correlation into the channel model.

\section{ACKNOWLEDGMENT}

This work was supported by the National Research Fund, Luxembourg under the CORE project " $\mathrm{CO}^{2} \mathrm{SAT}$ : Cooperative and Cognitive Architectures for Satellite Networks". The authors would like to thank Dr. Gan Zheng for useful discussions.

\section{REFERENCES}

[1] S. V. Hanly and P. A. Whiting, "Information-theoretic capacity of multireceiver networks," Telecommun. Syst., vol. 1, pp. 1-42, 1993.

[2] A. Wyner, "Shannon-theoretic approach to a Gaussian cellular multipleaccess channel," IEEE Trans. Inf. Theory, vol. 40, no. 6, pp. 1713-1727, Nov 1994.

[3] O. Somekh and S. Shamai, "Shannon-theoretic approach to a Gaussian cellular multiple-access channel with fading," IEEE Trans. Inf. Theory, vol. 46, no. 4, pp. 1401-1425, Jul 2000.

[4] S. Chatzinotas, M. Imran, and C. Tzaras, "On the capacity of variable density cellular systems under multicell decoding," IEEE Commun. Lett., vol. 12, no. 7, pp. 496 - 498, Jul 2008.

[5] S. Chatzinotas, M. A. Imran, and C. Tzaras, "Optimal information theoretic capacity of the planar cellular uplink channel," in IEEE 9th Workshop on Signal Processing Advances in Wireless Communications (SPAWC'08), Pernambuco, Brazil, Jul 2008, pp. 196-200.

[6] D. Aktas, M. Bacha, J. Evans, and S. Hanly, "Scaling results on the sum capacity of cellular networks with MIMO links," IEEE Trans. Inf. Theory, vol. 52, no. 7, pp. 3264-3274, July 2006.

[7] S. Chatzinotas, M. A. Imran, and C. Tzaras, "Uplink capacity of MIMO cellular systems with multicell processing," in IEEE International Symposium on Wireless Communication Systems (ISWCS'08), Reykjavik, Iceland, Oct 2008, pp. 453-457.

[8] V. Cadambe and S. Jafar, "Interference alignment and degrees of freedom of the $\mathrm{k}$-user interference channel," IEEE Trans. Inf. Theory, vol. 54 , no. 8 , pp. $3425-3441$, aug. 2008.

[9] — "Interference alignment and the degrees of freedom of wireless X networks," IEEE Trans. Inf. Theory, vol. 55, no. 9, pp. 3893 -3908, sept. 2009.

[10] S. Jafar and S. Shamai, "Degrees of freedom region of the MIMO X channel," IEEE Trans. Inf. Theory, vol. 54, no. 1, pp. $151-170$, jan. 2008.

[11] K. Gomadam, V. Cadambe, and S. Jafar, "Approaching the capacity of wireless networks through distributed interference alignment," in Global Telecommunications Conference, 2008. IEEE GLOBECOM 2008. IEEE, nov. 2008 , pp. $1-6$.

[12] C. M. Yetis, T. Gou, S. A. Jafar, and A. H. Kayran, "On feasibility of interference alignment in MIMO interference networks," IEEE Trans. Signal Processing, vol. PP, no. 99, pp. 1-1, 2010.

[13] R. Tresch, M. Guillaud, and E. Riegler, "On the achievability of interference alignment in the K-user constant MIMO interference channel," in Statistical Signal Processing, 2009. SSP '09. IEEE/SP 15th Workshop on, aug. 2009, pp. $277-280$.

[14] A. Goldsmith, S. Jafar, I. Maric, and S. Srinivasa, "Breaking spectrum gridlock with cognitive radios: An information theoretic perspective," Proceedings of the IEEE, vol. 97, no. 5, pp. 894 -914, may 2009.

[15] C. Suh and D. Tse, "Interference alignment for cellular networks," in Communication, Control, and Computing, 2008 46th Annual Allerton Conference on, 23-26 2008, pp. 1037 -1044.

[16] S. Chatzinotas and B. Ottersten, "Interference mitigation techniques for clustered multicell joint decoding systems," EURASIP Journal on Wireless Communications and Networking, Special Issue on Multicell Cooperation for Next Generation Communication Systems, vol. 132, 2011.

[17] A. T. A. Masucci and M. Debbah, "Asymptotic analysis of uplink interference alignment in ricean small cells," in Globecom 2011, Houston, Texas, USA, 2011.

[18] M. D. M. Maso, L. S. Cardoso and L. Vangelista, "Orthogonal precoder for lte small-cells networks," IEEE Journal on Selected Areas in Communications, 2011.

[19] S. Chatzinotas, M. Imran, and R. Hoshyar, "On the multicell processing capacity of the cellular MIMO uplink channel in correlated Rayleigh fading environment," IEEE Trans. Wireless Commun., vol. 8, no. 7, pp. 3704-3715, July 2009. 